\title{
Study on Energy Band-gap Calculation of $\mathrm{CuGaS}_{2}$ Liuyang $\mathrm{YU}^{\mathrm{a}}$, Yong $\mathrm{X \textrm {u } ^ { \mathrm { b } }}$, Kegao LIU $\mathrm{c}^{*}$
}

School of Materials Science and Engineering, Shandong Jianzhu University, Fengming Road, Jinan 250101, China

aylyang8806@126.com, bxuyong2612@gamil.com, cliukg163@163.com

*Corresponding author

Keywords: $\mathrm{CuGaS}_{2}$, Density Functional Theory, First-principle, Band-gap

Abstract: $\mathrm{CuGaS}_{2}$ as a ternary semiconductor with chalcopyrite structure has a direct bang-gap of $2.43 \mathrm{eV}$ at room temperature corresponding to the wavelength of $0.51 \mu \mathrm{m}$, it is a promising absorption layer for photovoltaic material. In this work, convergence of the plane wave cut-off energy and the K-mesh were carried out based on first-principle of density functional theory, using VASP package. Lattice constants were optimized by convergence parameters, and the energy band-gap under generalized gradient approximation was calculated by setting convergence parameters and optimized lattice constants. The results indicate that $\mathrm{CuGaS}_{2}$ has an energy band-gap of $0.784 \mathrm{eV}$. The calculating results play a guiding role in doping $\mathrm{Ga}$ to $\mathrm{CuInS}_{2}$ to adjust energy band-gap.

\section{Introduction}

In recent years, application of ternary chalcopyrite semiconductors in solar cell technology has aroused great interest, especially in $\mathrm{CuInS}_{2}$ and $\mathrm{CuGaS}_{2}$. Both $\mathrm{CuInS}_{2}$ and $\mathrm{CuGaS}_{2}$ are semiconductors with a direct band-gap, the band-gap of $\mathrm{CuInS}_{2}$ is $1.55 \mathrm{eV}$ and band-gap of $\mathrm{CuGaS}_{2}$ is $2.43 \mathrm{eV}$ [1-3].

First-principle calculations in the density functional theory, according to the principle of the atomic nucleus and electron interaction and the basic motion law, using quantum mechanics principle, basing on the specific requirements and a number of approximate treatment, it directly solves the Schrodinger equation to obtain the information of material structure and performance that we need [4].

Electronic structure and optical properties of $\mathrm{CuGaS}_{2}$ have been widely studied by experts and scholars in recent years. S. Laksariet et al calculated the band-gap of $\mathrm{CuGaS}_{2}$ as $0.903 \mathrm{eV}$ using a hybrid full-potential linear augmented plane-wave pluslocal orbitals (L/APW+lo) method [5], taking the local density approximation (LDA) for the exchange-correlation potentials. Amit Soniet et al separately used the linear combination of atomic orbitals (LCAO) and FP-LAPW method [6], the band-gaps value of $\mathrm{CuGaS}_{2}$ is $2.15 \mathrm{eV}$ based on LCAO-GGA while that from FP-LAPW method is $0.86 \mathrm{eV}$. Bin $\mathrm{Xu}$ et al figured out the band-gap of CGS as $0.818 \mathrm{eV}$ by using Wien $2 \mathrm{k}$ package, based on method of FPAPW [7].

In this work, we used the generalized gradient approximation as the exchange-correlation and carried out the convergence of the plane wave cut-off energy and K-mesh of $\mathrm{CuGaS}_{2}$ by using the VASP package [8]. The lattice constants of $\mathrm{CuGaS}_{2}$ were optimized according to the convergence parameters, thus the band-gap of $\mathrm{CuGaS}_{2}$ was calculated.

\section{Construction model}

It constructs the crystal cell model data taken from the ICSD database and reference [7], $\mathrm{CuGaS}_{2}$ is a ternary compound with chalcopyrite structure, which belongs to tetragonal system, whose corresponding space group number is 122, I-42d. The lattice constants of CGS were selected as $a=b=5.35 \AA, c=10.47 \AA, \alpha=\beta=\gamma=90^{\circ}$. The corresponding crystal structure model was built in MS as shown in Fig.1. 


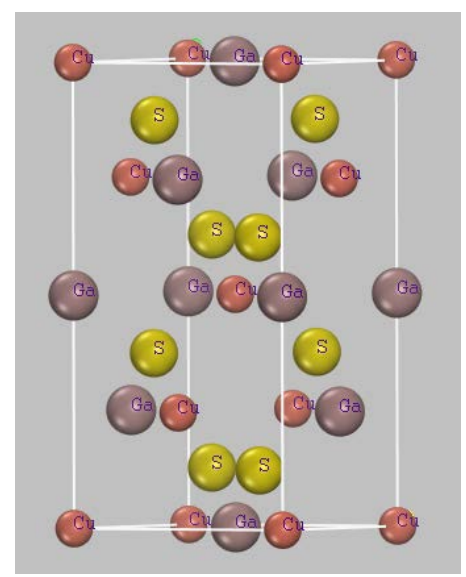

Fig. 1 The crystal structure of $\mathrm{CuGaS}_{2}$

\section{Parameters convergence and band-gap calculation}

Plane wave cut-off energy, K-mesh and lattice constants have effects on the calculation of the band-gap. So the convergence of the plane wave cut-off energy and K-mesh, and the optimization of the lattice constants were carried out firstly. After that, the band-gap of $\mathrm{CuGaS}_{2}$ can be calculated.

\section{Convergence of plane wave cut-off energy}

The convergence of plane wave cut-off energy was carried out under PBE pseudo potential of the generalized gradient approximation. K-mesh of $\mathrm{CuGaS}_{2}$ was chosen at $4 \times 4 \times 2,6 \times 6 \times 3,8 \times 8 \times 4$, $10 \times 10 \times 5,12 \times 12 \times 6$, then the parameters of plane wave cut-off energy were selected at $290 \mathrm{eV}, 300$ $\mathrm{eV}, 310 \mathrm{eV}, 320 \mathrm{eV}, 330 \mathrm{eV}, 340 \mathrm{eV}, 350 \mathrm{eV}$ and $360 \mathrm{eV}$. Running the calculation script, after the completion of the calculation to check the result (the difference between two energy value is less than $10^{-3} \mathrm{eV}$ ), it is found that $\mathrm{CuGaS}_{2}$ in the above K-mesh, all the convergence values are $340 \mathrm{eV}$. Plane wave cut-off energy convergence of $\mathrm{CuGaS}_{2}$ which selected as $6 \times 6 \times 3$ of $\mathrm{K}$-mesh was shown in Fig.2.

\section{Convergence of K-mesh}

K-mesh convergence of $\mathrm{CuGaS}_{2}$ was also carried out under PBE pseudo potential of the generalized gradient approximation. In the convergence of plane wave cut-off energy, we measured the value of $340 \mathrm{eV}$, and then the K-mesh was selected at $4 \times 4 \times 2,6 \times 6 \times 3,8 \times 8 \times 4,10 \times 10 \times 5$, $12 \times 12 \times 6$. Running the calculation script, after the completion of the calculation to check the results (the difference between two energy value is less than $10^{-3} \mathrm{eV}$ ), when the plane wave cut-off energy of $\mathrm{CuGaS}_{2}$ is $340 \mathrm{eV}$, the convergence of $\mathrm{K}$-mesh is $4 \times 4 \times 2$. The convergence of $\mathrm{K}$-mesh is shown in Fig.3. However, it exceeds the range of calculation when $\mathrm{K}$-mesh of $\mathrm{CuGaS}_{2}$ is chosen at $4 \times 4 \times 2$, so K-mesh of $\mathrm{CuGaS}_{2}$ is chosen at $6 \times 6 \times 3$.

\section{Optimization of lattice constants a and $b$}

After the convergence of plane wave cut-off energy and K-mesh were completed, the lattice constants of $\mathrm{CuGaS}_{2}$ need to be optimized. We fixed the position of atoms to optimize the lattice constants and volume of the unit cell. The optimization of lattice constant a (a=b) of $\mathrm{CuGaS}_{2}$ is

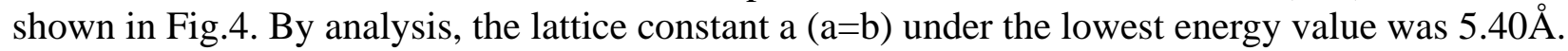




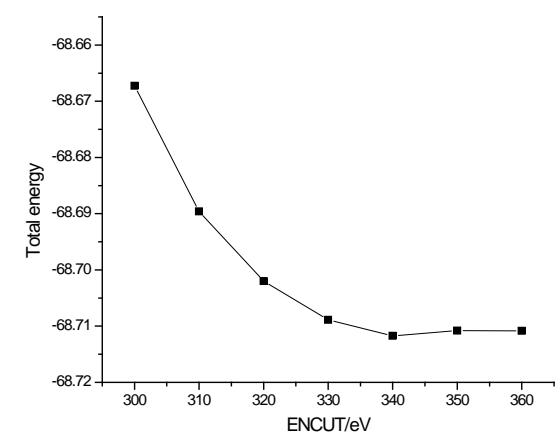

Fig. 2 The convergence of plane wave cut-off energy

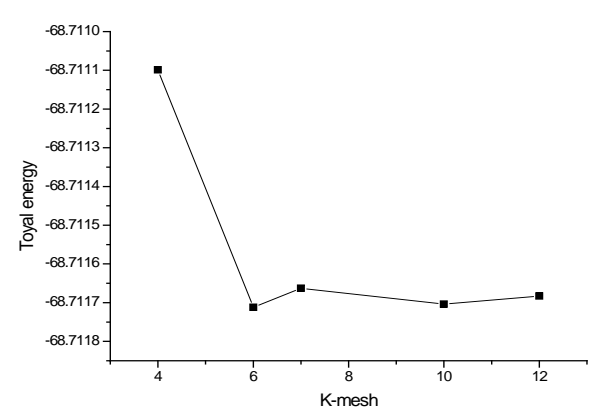

Fig.3 The convergence of K-mesh

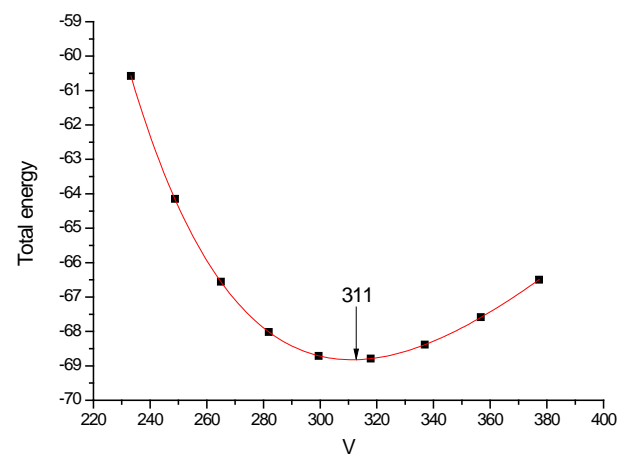

Fig. 4 The optimization of lattice constant a $(a=b)$

\section{Optimization of lattice constant c}

After optimization of the lattice constants a and b, we need to optimize the lattice constant c. Fixing the position of atoms to optimize the lattice constant and volume of the unit cell. The optimization of lattice constant $\mathrm{c}$ is shown in Fig.5. After analysis, the lattice constant $\mathrm{c}$ under the lowest energy value was $10.47 \AA$.

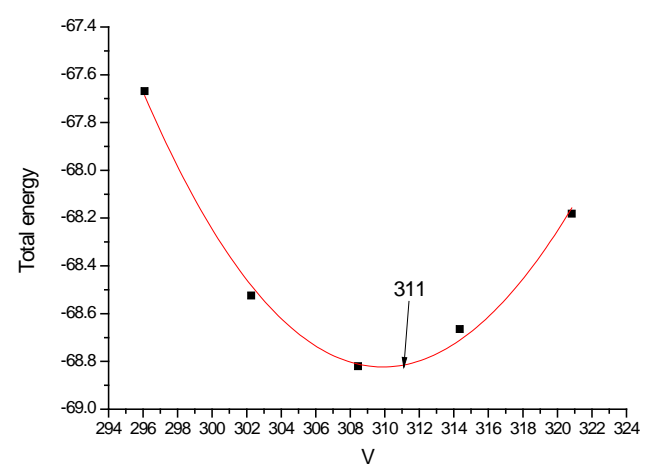

Fig. 5 The optimization of lattice constant c

\section{Calculated of band-gap}

The band-gap of $\mathrm{CuGaS}_{2}$ is calculated using VASP package, we need four files including INCAR, KPOINTS, POSCAR, POTCAR. In which the INCAR and KPOINTS parameters convergence have been completed, POSCAR file provides the atomic information and atomic positions that derived from the crystal model after optimizing the lattice constants, POTCAR is the pseudo potential file, which chooses the PBE pseudo potential of the generalized gradient approximation for calculation. Firstly, we carried out a static self-consistent calculation to get the correct charge density, then modified parameters to calculate band-gap. Here in the KPOINTS file we select the high symmetry point of the Brillouin zone, in this paper, Z, A, M, G, Z, R, X, G [9] were selected as high symmetry point through calculate and analysis, the energy band-gap diagram 
of the CGS is obtained as shown in Fig.6 [10]. It can be seen from Fig.6, the top of the valence bands and the bottom of the conduction bands lie along the Gamma indicating that $\mathrm{CuGaS}_{2}$ is a direct band-gap semiconductor, and band-gap is $0.784 \mathrm{eV}$, which has difference with the theoretical values. The calculated values are affected by lattice constants selection of pseudo potential and selection of exchange-correlation.

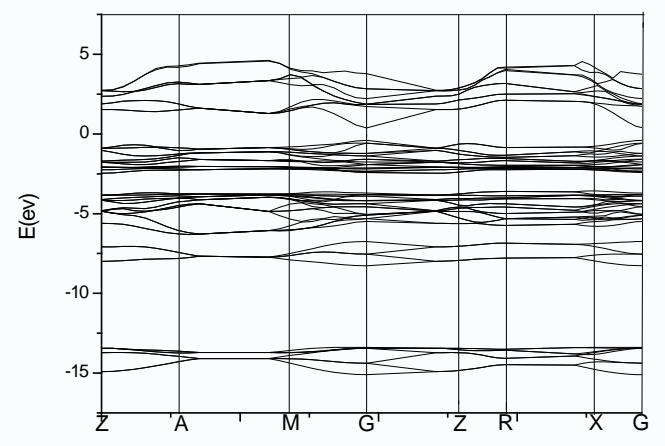

Fig. 6 Energy band diagram of $\mathrm{CuGaS}_{2}$

\section{Conclusions}

The band-gap of $\mathrm{CuGaS}_{2}$ was calculated based on first-principle of density functional theory, using VASP package. Through the convergence, the plane wave cut-off energy could be determined to be $340 \mathrm{eV}$, and K-mesh was $6 \times 6 \times 3$. The optimized lattice constant a $(\mathrm{a}=\mathrm{b})$ was $5.40 \AA$ and c was $10.47 \AA$. And then calculation of the $\mathrm{CuGaS}_{2}$ band-gap was carried out. It is shown that $\mathrm{CuGaS}_{2}$ is a direct band-gap semiconductor material and the band-gap is $0.784 \mathrm{eV}$. The calculating results of $\mathrm{CuGaS}_{2}$ energy band-gap play a guiding role in doping $\mathrm{Ga}$ to $\mathrm{CuInS}_{2}$ to adjust energy band-gap.

\section{Acknowledgment}

This work was financially supported by the National Natural Science Foundation of China (No.51272140).

\section{References}

[1] S.X. Zhou, L. Fang. Solar cells based on $\mathrm{CuInS}_{2}$ thin film. Solar thermal battery and its application, In Chinese, 2007 36(11) 848-852.

[2] J. L. Shay, P. M. Bridenbaugh, H.M. Kasper. Luminescent properties of $\mathrm{CuGaS}_{2}$ doped with Cd or Zn. Journal of Applied Physics, 1974 45(10) 4491-4494.

[3] P. Nayebi, K. Mirabbaszadeh, M. Shamshirsaz. Density functional theory of structural, electronic and optical properties of $\mathrm{CuXY}_{2}(\mathrm{X}=\mathrm{In}, \mathrm{Ga}$ and $\mathrm{Y}=\mathrm{S}$, Se) chalcopyrite semiconductors. Physica B, 416 (2013) 55-63.

[4] M. C. Payne, M. P. Teter, D. C. Allen, et al, Rev. Mod. Iterative minimization techniques for ab initio total-energy calculations: molecular dynamics and conjugate gradients. Phys, 199264 (4) 1045-1097

[5] S. Laksari, A. Chahed, N. Abbouni, et al. First-principles calculations of the structural, electronic and optical properties of $\mathrm{CuGaS} 2$ and $\mathrm{AgGaS}_{2}$. Computational Materials Science, 38 (2006) 223-230

[6] A. Soni, V. Gupta, C.M. Arora, et al. Electronic structure and optical properties of $\mathrm{CuGaS}_{2}$ and $\mathrm{CuInS}_{2}$ solar cell materials. Solar Energy, 84 (2010) 1481-1489

[7] B. Xu, X.F. Li, Z. Qin, et al. Electronic and optical properties of $\mathrm{CuGaS}_{2}$ : First-principles 
calculations. Physica B, 406 (2011) 946-951.

[8] G. Kresse, M. Marsman, J. Furthmüller. VASP the GUIDE. Vienna University, 2009.

[9] W. Setyawan, S. Curtarolo. High-throughput electronic band structure calculations: Challenges and tools. Computational Materials Science, 49 (2010) 299-312.

[10] Z.F. Hou. VASP the use of the software package Guide. Department of physics, Fudan University, 11 (2005) 28-29. 